PROCEEDINGS OF THE

AMERICAN MATHEMATICAL SOCIETY

Volume 132, Number 12, Pages 3679-3687

S 0002-9939(04)07497-0

Article electronically published on May 12, 2004

\title{
FIXED POINTS AND STABILITY OF A NONCONVOLUTION EQUATION
}

\author{
T. A. BURTON \\ (Communicated by Carmen C. Chicone)
}

ABSTRACT. In this note we consider an equation of the form

$$
x^{\prime}(t)=-\int_{t-r}^{t} a(t, s) g(x(s)) d s
$$

and give conditions on $a$ and $g$ to ensure that the zero solution is asymptotically stable. When applied to the classical case of $a(t, s)=a(t-s)$, these conditions do not require that $a(r)=0$, nor do they involve the sign of $a(t)$ or the sign of any derivative of $a(t)$.

\section{INTRODUCTION}

In this note we consider an equation which has attracted much attention during the last fifty years and has been studied by means of an intricate Liapunov functional. As a comparison to that work we present a simple stability and asymptotic stability result using a contraction mapping. In particular, we consider the scalar equation

$$
x^{\prime}(t)=-\int_{t-r}^{t} a(t, s) x(s) d s
$$

as well as the nonlinear analogue

$$
x^{\prime}(t)=-\int_{t-r}^{t} a(t, s) g(x(s)) d s,
$$

where it is assumed that $r$ is a positive constant, $a:[0, \infty) \times[-r, \infty) \rightarrow R$ is continuous,

$$
A(t, s):=\int_{t-s}^{r} a(u+s, s) d u \quad \text { with } \quad A(t, t)=\int_{0}^{r} a(u+t, t) d u \geq 0,
$$

and

$$
\beta:=\sup _{t \geq 0} \int_{0}^{r}\left|\int_{w}^{r} a(v+t-w, t-w) d v\right| d w<1 / 2 .
$$

Conditions (3) and (4) are sufficient for stability of (1); asymptotic stability requires that the integral of $A(t, t)$ diverge. For (2) it is supposed that $x g(x)>0$ for

Received by the editors July 8, 2003 and, in revised form, September 3, 2003.

2000 Mathematics Subject Classification. Primary 34K20, 47H10.

Key words and phrases. Delay equations, fixed points, stability.

(C)2004 American Mathematical Society 
small $x \neq 0$, plus other conditions including (3) and (4), to ensure stability and asymptotic stability.

\section{Some BACKGROUND}

Such equations have been the center of much interest for a very long time. In 1954 Brownell and Ergen [2] studied a form of (2) in connection with reactor dynamics. Nohel [10] picked up that work in 1960 and four years later was joined by Levin [9]. The previous year Levin had studied a related problem [7]

$$
x^{\prime}(t)=-\int_{0}^{t} a(t-s) g(x(s)) d s
$$

under the usual condition that $x g(x)>0$ for $x \neq 0$ plus the fairly severe condition that

$$
(-1)^{k} a^{(k)}(t) \geq 0, \quad 0<t<\infty, \quad k=0,1,2,3 .
$$

Levin states in that work [6, p. 535] that Volterra [11] had considered a linear version and suggested that a Liapunov functional might be constructed. Taking that suggestion, Levin constructed such a functional for (5) under conditions (6). Levin and Nohel [9, p. 35] note that with small changes Levin's functional works for

$$
x^{\prime}(t)=-\int_{t-r}^{t} a(t-s) g(x(s)) d s
$$

where $x g(x)>0$ for $x \neq 0$ and

$$
a(r)=0, \quad a(t) \geq 0, \quad a^{\prime}(t) \leq 0, \quad a^{\prime \prime}(t) \geq 0 \quad \text { for } \quad 0 \leq t \leq r .
$$

The Liapunov functional has the form

$$
V\left(x_{t}\right)=\int_{0}^{x} g(s) d s-\frac{1}{2} \int_{t-r}^{t} a^{\prime}(t-s)\left(\int_{s}^{t} g(x(v)) d v\right)^{2} d s
$$

where $x_{t}(s)=x(t+s)$ for $-r \leq s \leq 0$.

Using this Liapunov functional, Levin and Nohel [9, p. 35] are able to show that the zero solution of (7) is globally asymptotically stable and that some derivatives of the solution also tend to zero. Krasovskii [6, pp. 158-160] presents a well-organized list of conclusions concerning solutions of (7) under a variety of hypotheses. Hale 3 uses this Liapunov functional to great advantage in illustrating his theory of limit sets. Four years later, Hale 4 points out that with small modifications and convergence conditions Levin's functional still works for

$$
x^{\prime}(t)=-\int_{-\infty}^{t} a(t-s) g(x(s)) d s .
$$

Equation (7) with (8) and (9) became a classic and is featured in the texts by Krasovskii [6] pp. 158-160], Yoshizawa [12, pp. 198-199], and Hale [5, pp. 120-124]. It continues to be discussed today.

Levin [8] extended the work on (5) to the nonconvolution equation

$$
x^{\prime}(t)=-\int_{0}^{t} a(t, s) g(x(s)) d s
$$


asking that

$$
a(t, s) \geq 0, \quad a_{s}(t, s) \geq 0, \quad a_{s t}(t, s) \leq 0,
$$

and that work was extensively discussed in the subsequent literature. We find no corresponding extension of (7). Our present work is an effort in that direction using fixed point theory. Although the work presented here centers on the nonconvolution case, both linear and nonlinear, in the linear convolution case there is a result in [1, p. 162] based on a Liapunov functional that is better than the one presented here.

Our conditions (3) and (4) are independent of Levin's conditions (8), even in the convolution case. We are asking for a type of boundedness on $a(t, s)$, while (8) does not. On the other hand, our condition asks nothing at all about smoothness or the value of $a(r)$ even when restricted to the convolution case.

We noted above that the result of Levin and Nohel [9, p. 35] shows that both $x$ and several of its derivatives tend to zero, while ours does not mention the derivatives. However, it is clear from (1) that if $\int_{t-r}^{t}|a(t, s)| d s$ is bounded and if $x(t) \rightarrow 0$, then $x^{\prime}(t) \rightarrow 0$. One may differentiate (1) and argue that $x^{\prime \prime}(t) \rightarrow 0$. In the work cited in [9], investigators ask that $x g(x)>0$ for $x \neq 0$ and show that all solutions tend to zero. We only ask that $x g(x)>0$ for small $x \neq 0$ and show local asymptotic stability.

\section{THE LINEAR CASE}

We return to Equation (1) from Section 1, which we rewrite for reference as

$$
x^{\prime}(t)=-\int_{t-r}^{t} a(t, s) x(s) d s .
$$

Here, $r$ is a positive constant and $a:[0, \infty) \times[0, \infty) \rightarrow R$ is continuous.

Theorem 1. If (3) and (4) hold, then the zero solution of (1) is stable. If, in addition, $\int_{0}^{t} A(s, s) d s \rightarrow \infty$ as $t \rightarrow \infty$, then the zero solution of (1) is asymptotically stable.

Proof. We offer proofs only for $t_{0}=0$, and we cover only the case of the asserted asymptotic stability. Let $\psi:[-r, 0] \rightarrow R$ be a given continuous initial function, let $C$ be the set of continuous functions, and let

$$
M=\left\{\phi:[-r, \infty) \rightarrow R: \phi_{0}=\psi, \phi \in C, \phi(t) \rightarrow 0 \text { as } t \rightarrow \infty\right\}
$$

so that if $\|\cdot\|$ is the supremum metric, then $(M,\|\cdot\|)$ is a complete metric space. Here, $\phi_{0}=\psi$ means that $\phi(t)=\psi(t)$ for $-r \leq t \leq 0$. It will cause no confusion to also let $\|\psi\|$ denote the supremum of $\psi$ on $[-r, 0]$ even though we use $\|\phi\|$ as the supremum of $\phi$ on $[-r, \infty)$.

Write (1) as

$$
x^{\prime}(t)=-A(t, t) x(t)+\frac{d}{d t} \int_{t-r}^{t} A(t, s) x(s) d s .
$$


By the variation of parameters formula followed by integration by parts, we have

$$
\begin{aligned}
x(t) & =e^{-\int_{0}^{t} A(s, s) d s} \psi(0)+\int_{0}^{t} e^{-\int_{s}^{t} A(u, u) d u} \frac{d}{d s} \int_{s-r}^{s} A(s, u) x(u) d u d s \\
& =e^{-\int_{0}^{t} A(s, s) d s} \psi(0)+\left.e^{-\int_{s}^{t} A(u, u) d u} \int_{s-r}^{s} A(s, u) x(u) d u\right|_{s=0} ^{s=t} \\
& -\int_{0}^{t} e^{-\int_{s}^{t} A(u, u) d u} A(s, s) \int_{s-r}^{s} A(s, u) x(u) d u d s \\
& =e^{-\int_{0}^{t} A(s, s) d s} \psi(0)-e^{-\int_{0}^{t} A(s, s) d s} \int_{-r}^{0} A(0, u) \psi(u) d u \\
& +\int_{t-r}^{t} A(t, u) x(u) d u-\int_{0}^{t} e^{-\int_{s}^{t} A(u, u) d u} A(s, s) \int_{s-r}^{s} A(s, u) x(u) d u d s .
\end{aligned}
$$

Use (12) to define the operator $P: M \rightarrow M$ as follows: for $\phi \in M$, let $(P \phi)(t)=$ $\psi(t)$ if $-r \leq t \leq 0$, and if $t>0$, let

$$
\begin{aligned}
(P \phi)(t) & =e^{-\int_{0}^{t} A(s, s) d s} \psi(0)-e^{-\int_{0}^{t} A(s, s) d s} \int_{-r}^{0} A(0, u) \psi(u) d u \\
& +\int_{t-r}^{t} A(t, u) \phi(u) d u-\int_{0}^{t} e^{-\int_{s}^{t} A(u, u) d u} A(s, s) \int_{s-r}^{s} A(s, u) \phi(u) d u d s .
\end{aligned}
$$

A fixed point is a solution of (1).

Clearly, $\phi \in M$ implies that $P \phi$ is continuous. We can give the classical argument that the convolution of an $L^{1}$ function with a function tending to zero does itself tend to zero to show that $(P \phi)(t) \rightarrow 0$; use is made of the fact that $\int_{t-r}^{t}|A(t, u)| d u<$ $1 / 2$ by (4) using a change of variable.

To see that $P$ is a contraction, consider $\phi, \eta \in M$. For $t>0$, we have

$$
\begin{aligned}
|(P \phi)(t)-(P \eta)(t)| & \leq \int_{t-r}^{t}|A(t, u)(\phi(u)-\eta(u))| d u \\
& +\int_{0}^{t} e^{-\int_{s}^{t} A(u, u) d u} A(s, s) \int_{s-r}^{s}|A(s, u)(\phi(u)-\eta(u))| d u d s \\
& \leq \int_{t-r}^{t}|A(t, u)| d u\|\phi-\eta\|+\sup _{t \geq 0} \int_{t-r}^{t}|A(t, u)| d u\|\phi-\eta\| .
\end{aligned}
$$

It follows that $\|P \phi-P \eta\| \leq \alpha\|\phi-\eta\|$ for $t \in[-r, \infty)$, for some $\alpha>0$. Thus, $P$ is a contraction on $M$ if there is an $\alpha<1$ such that

$$
2 \sup _{t \geq 0} \int_{t-r}^{t}|A(t, u)| d u \leq \alpha .
$$

Observe that this does hold if $(4)$ is satisfied since the left-hand side of $\left(4^{*}\right)$ is $2 \beta$. This is easily seen with the change of variable $u=t-w$ in (4). In other words, under assumption (4), associated with each continuous initial function $\psi$ is a complete metric space $M$ and a unique $\phi \in M$ that is a fixed point of $P$. Equivalently, $\phi$ is the unique solution of $(1)$ with $\phi(t)=\psi(t)$ for $-r \leq t \leq 0$. 
We now want to find an $\epsilon-\delta$ relation for stability. If $\phi \in M$, then we have

$$
\begin{aligned}
|(P \phi)(t)| & \leq\|\psi\|\left(1+\int_{-r}^{0}|A(0, u)| d u\right)+2 \sup _{t \geq 0} \int_{t-r}^{t}|A(t, u)| d u\|\phi\| \\
& \leq\|\psi\|\left(1+\int_{-r}^{0}|A(0, u)| d u\right)+2 \beta\|\phi\|
\end{aligned}
$$

where $\beta$ is defined in (4). Since $P$ is a contraction, for each $\psi$ there is a unique $\phi$ with $P \phi=\phi$ so that

$$
\|\phi\|(1-2 \beta) \leq\|\psi\|\left(1+\int_{-r}^{0}|A(0, u)| d u\right) .
$$

Thus, we can find $\delta>0$ such that $\|\psi\|<\delta$ implies that $\|\phi\|<\epsilon$. Now $\phi$ is the unique solution and $\phi \in M$; so $\phi(t) \rightarrow 0$. Hence, $\phi_{t} \rightarrow 0$ as $t \rightarrow \infty$.

\section{A NONLINEAR EQUATION}

We return to Equation (2) from Section 1, which we rewrite for reference as

$$
x^{\prime}(t)=-\int_{t-r}^{t} a(t, s) g(x(s)) d s .
$$

Here, $r$ is a positive constant and $a:[0, \infty) \times[-r, \infty) \rightarrow R$ is continuous.

Remark. We assume that there is an $L>0$ such that $g$ is continuous, odd, Lipschitz, and increasing on $[-L, L]$ and that $0 \leq x-g(x)$ is nondecreasing on $[0, L]$. These conditions hold in case $\frac{d}{d x} g(x)$ is continuous and positive at $x=0$ by redefining $g$ and $a$. In this case, there is an $L>0$ for which $\frac{d}{d x} g(x)>0$ on $[-L, L]$, with a maximum of $D>0$. Write $a(t, s) g(x)=a(t, s) D \frac{g(x)}{D}$, and note that $0 \leq x-\frac{g(x)}{D}$ is nondecreasing.

Theorem 2. Assume that there is an $L>0$ such that $g$ is continuous, odd, Lipschitz, and increasing on $[-L, L]$. If (3) and (4) hold and if $0 \leq x-g(x)$ is nondecreasing on $[0, L]$, then the zero solution of (2) is stable.

Proof. Write (2) as

$$
\begin{aligned}
x^{\prime}(t) & =-A(t, t) g(x(t))+\frac{d}{d t} \int_{t-r}^{t} A(t, s) g(x(s)) d s \\
& =-A(t, t) x(t)+A(t, t)[x(t)-g(x(t))]+\frac{d}{d t} \int_{t-r}^{t} A(t, s) g(x(s)) d s .
\end{aligned}
$$

By the variation of parameters formula, followed by integration by parts, we have

$$
\begin{aligned}
x(t) & =e^{-\int_{0}^{t} A(s, s) d s} \psi(0)-e^{-\int_{0}^{t} A(s, s) d s} \int_{-r}^{0} A(0, u) g(\psi(u)) d u \\
& +\int_{0}^{t} e^{-\int_{s}^{t} A(u, u) d u} A(s, s)[x(s)-g(x(s))] d s+\int_{t-r}^{t} A(t, u) g(x(u)) d u \\
& -\int_{0}^{t} e^{-\int_{s}^{t} A(u, u) d u} A(s, s) \int_{s-r}^{s} A(s, u) g(x(u)) d u d s .
\end{aligned}
$$

Define

$$
M=\left\{\phi:[-r, \infty) \rightarrow R: \phi_{0}=\psi, \phi \in C,|\phi(t)| \leq L\right\}
$$


where $\psi$ will be restricted in magnitude later. Use (14) to define $P: M \rightarrow M$ by $\phi \in M$ implies that $(P \phi)(t)=\psi(t)$ if $-r \leq t \leq 0$, and if $t \geq 0$, then

$$
\begin{aligned}
(P \phi)(t) & =e^{-\int_{0}^{t} A(s, s) d s} \psi(0)-e^{-\int_{0}^{t} A(s, s) d s} \int_{-r}^{0} A(0, u) g(\psi(u)) d u \\
& +\int_{0}^{t} e^{-\int_{s}^{t} A(u, u) d u} A(s, s)[\phi(s)-g(\phi(s))] d s+\int_{t-r}^{t} A(t, u) g(\phi(u)) d u \\
& -\int_{0}^{t} e^{-\int_{s}^{t} A(u, u) d u} A(s, s) \int_{s-r}^{s} A(s, u) g(\phi(u)) d u d s .
\end{aligned}
$$

Since $g$ is increasing and $x-g(x)$ is nondecreasing on $[0, L]$ and odd, we have

$$
\begin{aligned}
|(P \phi)(t)| & \leq\|\psi\|+\|g(\psi)\| \int_{-r}^{0}|A(0, u)| d u \\
& +L-g(L)+2 g(L) \sup _{t \geq 0} \int_{t-r}^{t}|A(t, u)| d u \\
& =\|\psi\|+\|g(\psi)\| \int_{-r}^{0}|A(0, u)| d u+L-g(L)+2 \beta g(L)
\end{aligned}
$$

where $\beta$ is defined in (4). By (4) again, we can find $\alpha<1$ with $L-g(L)+2 \beta g(L)=$ $\alpha L$. Since $g$ is Lipschitz and $g(0)=0$, there is a $K$ with $|g(\psi(t))| \leq K|\psi(t)|$. Thus, we can find $\delta>0$ such that $\|\psi\|<\delta$ implies that

$$
\|\psi\|+\|g(\psi)\| \int_{-r}^{0}|A(0, u)| d u<(1-\alpha) L .
$$

This shows that $P: M \rightarrow M$.

In the proposition below we show that there is an exponentially weighted metric under which $P$ is a contraction with unique fixed point $\phi$ for each $\psi$.

To see that we have proved stability, for a given $\epsilon>0, \epsilon<L$, substitute $\epsilon$ for $L$ in the above argument and conclude that $\|\psi\|<\delta$ yields $|(P \phi)(t)| \leq \epsilon$; if $P \phi=\phi$, then $|\phi(t)| \leq \epsilon$.

Remark. We only conclude stability, not asymptotic stability here, even if we ask that the integral of $A(t, t)$ diverges. The reason for that lies in our change of metric. If we were to add the condition to $M$ that $\phi(t) \rightarrow 0$, then under our new metric, $M$ would no longer be a complete metric space.

Proposition. Under the conditions of Theorem 2, there is a metric $\rho$ such that $(M, \rho)$ is a complete metric space and $P: M \rightarrow M$ is a contraction in that space.

Proof. Let $B:[0, \infty) \rightarrow[0, \infty)$ be defined by

$$
B(s):=\int_{0}^{r}|a(u+s, s)| d u,
$$

and let $d>3$. Denote by $K$ the common Lipschitz constant for $g(x)$ and $x-g(x)$ on $[-L, L]$. Define

$$
h(t)=d K \int_{0}^{t}[A(u, u)+B(u)] d u
$$


and define a metric $\rho$ on $M$ by $\phi, \eta \in M$ implies

$$
\rho(\phi, \eta)=|\phi-\eta|_{K}:=\sup _{0 \leq t<\infty}|\phi(t)-\eta(t)| e^{-h(t)}
$$

Then $(M, \rho)$ is a complete metric space.

Let $\phi, \eta \in M$ and use (14) to obtain

$$
\begin{aligned}
& |(P \phi)(t)-(P \eta)(t)| e^{-h(t)} \\
& \leq \int_{0}^{t} e^{-\int_{s}^{t} A(u, u) d u} A(s, s) K|\phi(s)-\eta(s)| e^{-h(t)+h(s)-h(s)} d s \\
& +\int_{t-r}^{t}|A(t, u)| K|\phi(u)-\eta(u)| e^{-h(t)+h(u)-h(u)} d u \\
& +\int_{0}^{t} e^{-\int_{s}^{t} A(u, u) d u} A(s, s) \int_{s-r}^{s}|A(s, u)| K|\phi(u)-\eta(u)| e^{-h(t)+h(u)-h(u)} d u d s \\
& \leq \int_{0}^{t} e^{-(d K+1) \int_{s}^{t} A(u, u) d u} A(s, s) K|\phi(s)-\eta(s)| e^{-h(s)} d s \\
& +\int_{t-r}^{t} e^{-d K \int_{u}^{t} B(s) d s}|A(t, u)| K|\phi(u)-\eta(u)| e^{-h(u)} d u \\
& +\int_{0}^{t} e^{-\int_{s}^{t} A(u, u) d u} A(s, s) \int_{s-r}^{s} e^{-d K \int_{u}^{s} B(v) d v}|A(s, u)| K|\phi(u)-\eta(u)| e^{-h(u)} d u d s \\
& \leq|\phi-\eta|_{K} K\left[\int_{0}^{t} e^{-(d K+1) \int_{s}^{t} A(u, u) d u} A(s, s) d s+\int_{t-r}^{t} e^{-d K \int_{u}^{t} B(s) d s}|A(t, u)| d u\right. \\
& \left.+\int_{0}^{t} e^{-\int_{s}^{t} A(u, u) d u} A(s, s) \int_{s-r}^{s} e^{-d K \int_{u}^{s} B(v) d v}|A(s, u)| d u d s\right] .
\end{aligned}
$$

For each fixed $t$, notice that in

$$
\int_{t-r}^{t} e^{-d K \int_{u}^{t} B(s) d s}|A(t, u)| d u
$$

we have $|A(t, u)| \leq \int_{0}^{r}|a(v+u, u)| d v=B(u)$ so that

$$
\int_{t-r}^{t} e^{-d K \int_{u}^{t} B(s) d s}|A(t, u)| d u \leq \int_{t-r}^{t} e^{-d K \int_{u}^{t} B(s) d s} B(u) d u \leq \frac{1}{d K}
$$

This also applies to the last integral in our array, and we arrive at

$$
\begin{aligned}
& |(P \phi)(t)-(P \eta)(t)| e^{-h(t)} \\
& \leq K|\phi-\eta|_{K}\left[\frac{1}{d K+1}+\frac{1}{d K}+\frac{1}{d K}\right] \\
& \leq \frac{3}{d}|\phi-\eta|_{K}
\end{aligned}
$$

which is a contraction since $d>3$. This completes the proof.

In our next result we abuse notation by letting $g^{\prime}(x)=\frac{d}{d x} g(x)$.

Corollary. Let the conditions of Theorem 2 hold, and suppose that $\int_{0}^{t} A(s, s) d s \rightarrow$ $\infty$ as $t \rightarrow \infty$. If $g^{\prime}(x)$ is continuous on $(-L, L)$ and if $g^{\prime}(0) \neq 0$, then the zero solution of (2) is asymptotically stable. 
Proof. It can be seen that if Theorem 2 holds for one $L>0$, then it holds for all smaller positive $L$. For a fixed $L>0$ of Theorem 2, let $q=\min g^{\prime}(x)$ and $Q=\max g^{\prime}(x)$ for $-L \leq x \leq L$. Since $2 \beta<1$ by (4), if $L$ is sufficiently small, then $q, Q$ will satisfy $2 \beta Q<q$. That is, as $L \rightarrow 0$, we may let $q, Q \rightarrow g^{\prime}(0)$. This means that

$$
\mu:=(1-q)+2 \beta Q<1
$$

For $L, q, Q$ chosen so that (16) holds, define

$$
M=\left\{\phi:[-r, \infty) \rightarrow R: \phi_{0}=\psi, \phi \in C,|\phi(t)| \leq L, \phi(t) \rightarrow 0\right\} .
$$

We have $P$ defined in (15), and it readily follows that $\phi \in M$ implies $(P \phi)(t) \rightarrow 0$. So $P: M \rightarrow M$.

To see that $P$ is a contraction using the supremum norm, for $f(x)=x-g(x)$ on $(-L, L)$ we have $0 \leq f^{\prime}(x)=1-g^{\prime}(x) \leq 1-q$ since by hypothesis, $f$ is increasing. We also have $0 \leq g^{\prime}(x) \leq Q$. Thus, for $\phi, \eta \in M$ we obtain

$$
\begin{aligned}
& |(P \phi)(t)-(P \eta)(t)| \\
& \leq \int_{0}^{t} e^{-\int_{s}^{t} A(u, u) d u} A(s, s)|f(\phi(s))-f(\eta(s))| d s \\
& \quad+\int_{t-r}^{t} \mid A(t, u)(g(\phi(u))-g(\eta(u)) \mid d u \\
& +\int_{0}^{t} e^{-\int_{s}^{t} A(u, u) d u} A(s, s) \int_{s-r}^{s} \mid A(s, u)(g(\phi(u))-g(\eta(u)) \mid d u d s \\
& \leq \\
& {\left[(1-q)+2 \sup _{t \geq 0} \int_{t-r}^{t}|A(t, u)| d u Q\right]\|\phi-\eta\|} \\
& \leq \\
& =\mu(1-q)+2 \beta Q]\|\phi-\eta\| \\
& =\mu\|\phi-\eta\| .
\end{aligned}
$$

This completes the proof.

\section{REFERENCES}

1. Burton, T. A. and Mahfoud, W. E., Stability criteria for Volterra equations, Trans. Amer. Math. Soc. 279 (1983), 143-174. MR 84h:45004

2. Brownell, F. H. and Ergen, W. K., A theorem on rearrangements and its application to certain delay differential equations, J. Rational Mech. Anal. 3(1954), 565-579. MR 16:714c

3. Hale, Jack, Sufficient conditions for stability and instability of autonomous functionaldifferential equations, J. Differential Equations 1(1965), 452-482. MR 32:1414

4. Hale, Jack, Dynamical systems and stability, J. Math. Anal. Appl. 26(1969), 39-59.

5. Hale, Jack, Theory of Functional Differential Equations, Springer, New York, 1977. MR 58:22904

6. Krasovskii, N. N., Stability of Motion, Stanford Univ. Press, Stanford, CA, 1963. MR 26:5258

7. Levin, J. J., The asymptotic behavior of the solution of a Volterra equation, Proc. Amer. Math. Soc. 14(1963), 534-541. MR 27:2824

8. Levin, J. J., A nonlinear Volterra equation not of convolution type, J. Differential Equations 4(1968), 176-186. MR 37:712

9. Levin, J. J. and Nohel, J. A., On a nonlinear delay equation, J. Math. Anal. Appl. 8(1964), 31-44. MR 29:445

10. Nohel, J. A., A class of nonlinear delay differential equations, J. Math. Physics 38(1960), 295-311. MR 22:4931 
11. Volterra, V., Sur la théorie mathématique des phénomènes héréditaires, J. Math. Pures Appl. 7(1928), 249-298.

12. Yoshizawa, T., Stability Theory by Liapunov's Second Method, Math. Soc. Japan, Tokyo, 1966. MR 34:7896

Northwest Research Institute, 732 Caroline St., Port Angeles, Washington 98362 E-mail address: taburton@olypen.com 